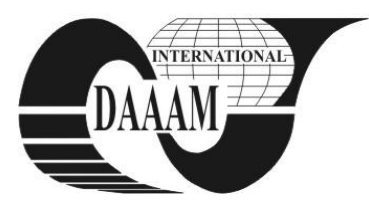

Annals of DAAAM for 2011 \& Proceedings of the 22nd International DAAAM Symposium, Volume 22, No. 1, ISSN 1726-9679 ISBN 978-3-901509-83-4, Editor B. Katalinic, Published by DAAAM International, Vienna, Austria, EU, 2011 Make Harmony between Technology and Nature, and Your Mind will Fly Free as a Bird Annals \& Proceedings of DAAAM International 2011

\title{
DYNAMIC MODELING OF THE CUTTING PROCESS
}

\author{
MADISSOO, M[arten] \& OLT, J[ueri]
}

\begin{abstract}
By mechanical processing of materials is critical to assessing the quality of processing accuracy. Hereby we propose a method for the construction of dynamic models of small dimensionality. On which the accomplished simulation of the technological systems of mechanical processing can be based. It is necessary for increasing the manufacturing precision.

Key words: metal machining, deformation, cutting, technological system
\end{abstract}

\section{INTRODUCTION}

For the definition of the characteristics reflecting the dynamic quality of a technological system it is necessary to construct the boundaries of its region of stability in the cutting process. This includes the construction of the dynamic model for a technological system that is a closed potentially autooscillatory system allowing for the maximum possible completeness of inert and elastic-dissipative properties in the technological system. The passage to a simplified dynamic model can be in comparing the equivalent to the relatively spectral characteristics of the initial one, applying the substantiated criterion of proximity in the assigned frequency range. Construction in the space of parameters of the following limits for the region of stability: a) upper maximum - built on the assumption that the workpiece is absolutely solid; b) lower maximum - built on the use of static characteristics in where the system is basing on the workpiece fastening. The relative position of the upper and the lower maximum boundaries determines the reserve for an increase in the dynamic quality to perform control of the technological system.

\section{MATERIALS AND METHODS}

The properties of a technological system are defined by its static and dynamic characteristics. The most important static characteristics are the stiffness of the carrying systems, their geometric accuracy and the errors in sizes and form determined by them in the quasi-static regimes of working. Dynamic characteristics determine the zones of stability of the cutting process, the spectral composition and level of amplitudes of oscillating processes, dynamic errors in sizes and form of products, and also the properties of the surface layer (Matalin, 1985; Stephenson, 2006).

As a rule, it is the intuitive-topological method that is used for the development of models of carrier systems. The essence of this method consists in the disassembly of the carrier system into separate inert blocks, which correspond to the models of an absolute solid. It is assumed that the blocks are interconnected with elastic joints. The totality of such subsystems of the carrier system forms a three-dimensional and according to some assumptions - a flat dynamic model of the system (Murashkin, 1977; Atkins, 2003).

Knowing that the elastic and dissipative properties of a technological system are determined in essence by deformations and damping of joints, it is possible to devise a simplified dynamic model of a machine tool for practical calculations (Panovko, 1960). Furthermore, with the composition of the dynamic model of a technological system according to the basic concept of the vibration theory (Vlado, 2002; Panovko, 1960), it is possible to limit the degrees of freedom to a finite number when the dynamic properties of a system are examined in the limited range of frequency. The basic parameters of the discrete dynamic model of an oscillatory system include (Murashkin, 1977; Atkins, 2003): reduced masses $m$, stiffness coefficients $c$, coefficients of force $b$ and of moments $b_{\varphi}$ of linearized resistances. For determining the parameters indicated above the axes of the three-dimensional system of coordinates for the process of grinding were oriented as follows: $z$ axis the direction of longitudinal feed, axis $x$ cross feed and $y$ axis is perpendicular to plane $x-z$. According to the studies (Murashkin, 1977; Atkins; 2003; Panovko, 1960), carried out in the field of the vibration theory of technological systems, it is possible to assume that the fluctuations in different directions are not interrelated.

The value of reduced mass $i$ - of that element of a technological system $m_{i}$ is determined from the formula (4)

$$
m_{i}=\frac{c_{i}}{\omega_{i}^{2}}
$$

where $c_{i}$ - stiffness coefficient, N/m; $\boldsymbol{\omega}_{\boldsymbol{i}}$ - the intrinsic angular frequency, $\mathrm{s}^{-1}$. Force coefficient of the linearized resistance $b_{i}$ $(\mathrm{N} \cdot \mathrm{s} / \mathrm{m})$ is equal to $(4)$

$$
b_{i}=2 \cdot \lambda_{i} \cdot f_{c i} \cdot m_{i}
$$

where $\lambda_{i}$ - logarithmic decrement is $i$-th element of the system; $f_{c i}$ - the natural frequency of the element, Hz. For the torsional system the given moment of inertia is determined $J_{\varphi}\left(\mathrm{kg} \cdot \mathrm{m}^{2}\right)$ (4)

$$
J_{\varphi}=\frac{c_{\varphi}}{\omega_{\varphi}^{2}}
$$

where $c_{\varphi}-$ the coefficient of torsional stiffness of the element, $\mathrm{N} \cdot \mathrm{m} / \mathrm{rad} ; \boldsymbol{\omega}_{\varphi}-$ the angular frequency of the natural torsional oscillations, $\mathrm{s}^{-1}$. Coefficient of the moment of linearized resistance $b_{\varphi}(\mathrm{N} \cdot \mathrm{m} \cdot \mathrm{s})$ is determined from the formula (4)

$$
b_{\varphi}=2 \cdot \lambda_{\varphi} \cdot f_{\varphi} \cdot J_{\varphi}
$$

where $\lambda_{\varphi}-$ the logarithmic decrement of the natural torsional oscillations; $f_{\varphi}$ - the natural frequency of torsional oscillations, $\mathrm{Hz}$.

\section{THEORETICAL RESULTS}

For the analysis of the dynamic properties of a technological system it is necessary to switch over from the complex technological system to a simplified model. The dynamic model of a technological system can be represented as a complex system, which is organized hierarchically and is goal-directed to function as the totality of a large number of elements connected in terms of information and interaction (Murashkin, 1977; Panovko, 1960)

Using the concept of the delay of the cutting force with respect to the normal displacement for the description of dynamic properties of the cutting process, the dynamic characteristic of cutting can be written down in the following form (Vlado, 2002) 


$$
T_{P} \cdot \dot{P}+P=-k \cdot u_{P}
$$

where $P$ - the dynamic cutting force; $T_{p}$ - the time constant of delay; $k$ - the coefficient of disturbance; $u_{p}-$ a change in the area of shear.

The need for a passage from the global model of a technological system to the simplified model, is built by us according to the hierarchical principle, is accounted by the following basic considerations:

1. With the contemporary facilities of computer technology the structural complexity of the model and the large number of generalized coordinates necessary to achieve the discreteness of the system on the basis of the Finite Element Methods (FEM) is not the principal moment, constituting the need for a passage to simplified models.

2. In the limited frequency range the initial global model of a technological system can be infinitely closely represented by the simplified dynamic model with the appropriate identity of amplitude-phase-frequency characteristics (APFC). If we limit ourselves to the approximate construction of the limits of the region of stability in the space of parameters of the system on the basis of the first method of Lyapunov, then the simplified model, built on the basis of the principle indicated, will prove to be completely correct for finding the solution to the optimization problem by the criterion of maximum productivity of mechanical processing with corresponding limitations. Such natural constraints include durability of the cutting tool, dynamic accuracy and quality coefficients of the surface layer.

Taking into account the above, we consider that the motion of the simplified model of the technological system of mechanical processing can be represented by the system of differential equations of $m$-th order in the form

$$
\begin{gathered}
\dot{q}=D \cdot q+G \cdot U \\
y=C \cdot q
\end{gathered}
$$

where $q-m$ - the measured vector function of the generalized coordinates of the system; $y-k-$ the measured vector function of the outputs of the system; $U-l-$ the measured vector function of disturbances; $D-(m \times m)-$ the matrix of the coefficients of the simplified model; $G-(m \times l)$ - and $C-(k \times m)-$ the assigned matrices.

Applying to the system of equations (6) and (7) the direct one-sided Laplace transform with the zero initial conditions, we will obtain the transmitting matrix of the simplified model in the form

$$
\tilde{W}(p)=C \cdot(E \cdot p-D)^{-1} \cdot B
$$

where $\tilde{W}(p)-(k \times l)-$ the matrix; $E-(m \times m)-$ the unit matrix.

Assuming $p=j \cdot \omega_{i}$, we will obtain the matrix of the frequency characteristics of the simplified model above the field of complex numbers, the elements of which are determined by the following dependencies

$$
\begin{gathered}
\tilde{w}_{u, v}\left(j \cdot \omega_{i}\right)=m_{u, v}\left(\omega_{i}\right) \cdot \exp \left(j \cdot \varphi_{u, v}\right) \\
u=1,2, \ldots, k ; v=1,2, \ldots, l,
\end{gathered}
$$

where $m_{u, v}\left(\omega_{i}\right)=\left|\tilde{w}_{u, v}\left(j \cdot \omega_{i}\right)\right| ; \varphi_{u, v}\left(\omega_{i}\right)=\operatorname{Arg} \tilde{w}_{u, v}\left(j \cdot \omega_{i}\right)$.

Matrices $M\left(\omega_{i}\right)$ with the elements $m_{u}, \Phi\left(\omega_{i}\right)$ and $\varphi_{u, v}\left(\omega_{i}\right)$ with the elements of $\varphi_{u, v}\left(\omega_{i}\right)$ are called the matrix of amplitudefrequency and the matrix of phase responses (AFR and APR), respectively. Consequently, we offer two methods approach.

Determination 1 . Two systems are called equivalent, if the following condition is satisfied

$$
\rho(W, \tilde{W}) \leq \varepsilon
$$

where $\varepsilon$ - the small predetermined quantity. Fulfillment condition (10) ensures the proximity AFR and APR of the generalized and simplified models.
Determination 2. The simplified model with the matrix of coefficients $D^{*}$ of sizes $(m \times l)$ and the corresponding matrix of the frequency characteristics $\tilde{W}^{*}$ is called the optimum model of the $m$-th order, if the following condition is satisfied

$$
\rho\left(W, \tilde{W}^{*}\right)=\min \rho(W, \tilde{W})
$$

On the basis of definitions 1 and 2 we assumed that the simplified model of system with the matrix of coefficients $D^{*}$ is the optimum equivalent model of $m$-th order, if the following condition is satisfied

$$
\rho\left(W, \tilde{W}^{*}\right) \leq \varepsilon
$$

The analytical solution of the problem of finding the optimum equivalent model in the general case is impossible. Therefore, it is expedient to perform its construction by the numerical methods of search. It is recommended to start the process of search for the optimum equivalent model with the simplest singlemass two-circuit model. If in this case conditions (10), (12) are not satisfied, it is necessary to pass on to models of more complex structure.

It is proposed to use a four-contour model of small dimensionality with dissipative characteristics, which account for the structural damping and rheological processes of the global model in question, to which the two subsystems with four generalized coordinates correspond:

1 - the subsystem of workpiece B with the coordinates $u, w$; 2 - the subsystem of tool T with the coordinates $x, y$.

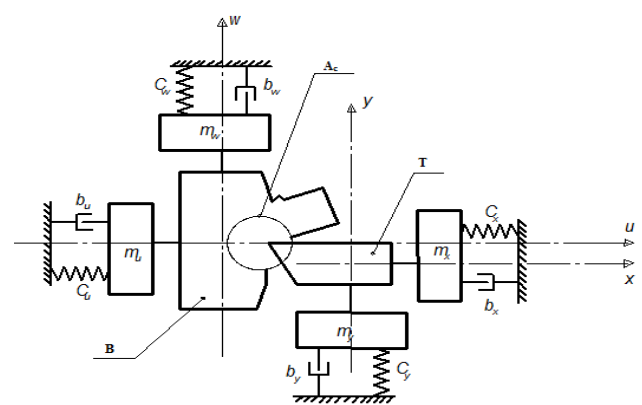

Fig. 1. The dynamic model of the four-contour technological system: B - subsystem "workpiece"; T - tool; $\mathrm{A}_{\mathrm{c}}$ - the operator of chip formation

\section{CONCLUSIONS}

We propose a procedure for the construction of a simplified dynamic model of a technological system as a closed potentially auto-oscillatory system allowing for a maximum possible completeness of inert and elastic-dissipative properties. The model helps to find stable machining parameters and therefore it is possible to improve the quality of manufactured parts. The downside is that the model is suitable for a limited range of data, so further work is planned to focus on suiting the model for the processing of heat resistant materials.

\section{REFERENCES}

Atkins A.G. (2003). Modelling metal cutting using modern ductile fracture mechanics: quantitative explanations for some longstanding problems, International Journal of Mechanical sciences 45 , pp. $373-396$

Matalin A.A. (1985). Mechanical engineering technology. Leningrad, Mashinostroenije, - 496. p. (in Russian)

Murashkin L.S., Murashkin S.L. (1977). Applied Nonlinear mechanics tools. L., Mashinostroenije, - 192 p. (in Russian)

Panovko Y.G. (1960). The internal friction vibrations of elastic systems. Moscow: Fizmatgiz. - 193 p. (in Russian)

Stephenson, David A., John S. (2006). Metal cutting theory and practice 2 nd. ed. by Taylor \& Francis Group, LLC, 825 p.

Vlado A. Lubarda. (2002). Elastoplasticity theory CRC Press, $387 \mathrm{p}$ 